\title{
Rapid Creation of Three-Dimensional, Tactile Models from Crystallographic Data
}

\author{
Nathan B. Fisher, John C. Charbonneau, and Stephanie K. Hurst \\ Department of Chemistry and Biochemistry, Northern Arizona University, P.O. Box 5698, Flagstaff, AZ 86011-5698, USA \\ Correspondence should be addressed to Stephanie K. Hurst; stephanie.hurst@nau.edu
}

Received 4 March 2016; Revised 30 June 2016; Accepted 4 July 2016

Academic Editor: Rajesh G. Gonnade

Copyright (C) 2016 Nathan B. Fisher et al. This is an open access article distributed under the Creative Commons Attribution License, which permits unrestricted use, distribution, and reproduction in any medium, provided the original work is properly cited.

\begin{abstract}
A method for the conversion of crystallographic information framework (CIF) files to stereo lithographic data files suitable for printing on three-dimensional printers is presented. Crystallographic information framework or CIF files are capable of being manipulated in virtual space by a variety of computer programs, but their visual representations are limited to the two-dimensional surface of the computer screen. Tactile molecular models that demonstrate critical ideas, such as symmetry elements, play a critical role in enabling new students to fully visualize crystallographic concepts. In the past five years, major developments in threedimensional printing has lowered the cost and complexity of these systems to a level where three-dimensional molecular models may be easily created provided that the data exists in a suitable format. Herein a method is described for the conversion of CIF file data using existing free software that allows for the rapid creation of inexpensive molecular models. This approach has numerous potential applications in basic research, education, visualization, and crystallography.
\end{abstract}

\section{Introduction}

Visualization has long been an indicator of success in chemistry education and has direct application to chemistry topics including chirality and symmetry [1]. Students initially visualize molecular structure through the use of molecular models before progressing to a purely mental construction of a molecule or unit cell. Molecular models have an extensive history in chemistry and have been in use long before the existence of atoms was firmly established. Models have been made out of materials as diverse as croquet balls and brass strips and in some cases may even be considered as works of art (e.g., Miramodus molecular models). However, from an educational perspective, traditional model kits have many limitations including a limited number of atoms of fixed geometry. From a crystallographic perspective, this is particularly problematic as models may not necessarily reflect accurate bond lengths and bond angles and coordination geometries or may be prohibitively expensive. From the perspective of an inorganic chemist, most model kits are not able to represent unusual coordination numbers or coordination geometry. Other topics that benefit greatly from the use of models include the structure of crystalline units cells, demonstration of the delta $(\Delta)$ and lambda $(\Lambda)$ isomers of octahedral metal complexes such as $\left[\mathrm{Ru}(\mathrm{phen})_{3}\right]^{2+}[2]$, and the symmetry properties of molecules of $D_{n}$ and $C_{n}$ point groups. The expense and limitations of molecular models to demonstrate critical learning concepts are even more pronounced at smaller institutions, particularly twoyear institutions and tribal colleges where funds may simply not be available to purchase and/or replace model kits every year for all students. Tactile models that go beyond those available through standard kits are also of great potential value to students who are either blind or visually impaired [3-5].

Given the importance of visualization and the limitation of existing model kits, one potential solution is through the use of the developing area of rapid prototyping techniques for molecular models. Rapid prototyping can be defined as a set of techniques used to quickly design a scale model of a part or assembly using three-dimensional Computer Aided Design (CAD) software followed by Computer Aided Manufacturing (CAM). For example, rapid prototyping has been used for the creation of microfluidic systems from poly(dimethyl 


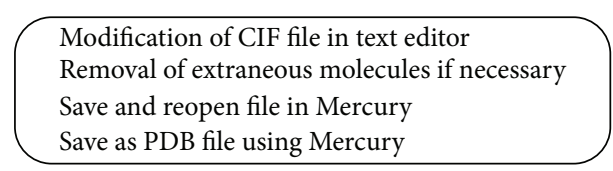

(a)

Open STL file in Netfabb Studio Basic
Invert surface normals if necessary
Repair holes and inverted triangles
Export part as STL file

(c)

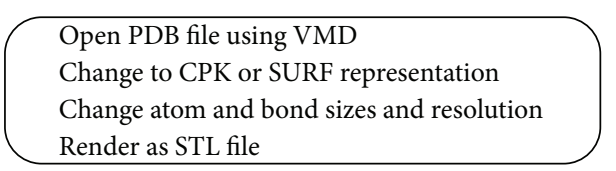

(b)

Open STL file in printer software
Determine fill ratio and amount of support
Print model at appropriate resolution and size
Remove support material

(d)

FIgure 1: Workflow diagram. (a) Preparation and modification of the existing crystallographic data. (b) Optimization of the PDB file and rendering as an STL file. (c) Repair of the STL file. (d) Final printing of the STL file as a molecular model.

siloxane) (PDMS) [6]. Several groups have already used rapid prototyping tools to develop tactile models for use in biochemistry education [7]. Production of the prototype parts is centred on the principle of three-dimensional (3D) printing, where three-dimensional printers or "fabricators" operate by laying down material and, through successive passes, build up a fully three-dimensional object. Many moderate-resolution printers now typically cost less than the desktop computer required to operate them and, in addition commercial firms, now offer "print on demand" services for three-dimensional objects. These commercial companies generally specialize in very high-quality structures, but purchasing sufficient quantities of these models for even a small lecture course would be prohibitive. Although three-dimensional printers have limitations, they are capable of printing molecules with vastly greater number of atoms than traditional model kits. In addition, our group has also used three-dimensional printers to construct parts that augment existing model kits [8, 9]. Thus, this new capability augments, enhances, and extends traditional models.

In recent years there have been many papers detailing the educational and scientific utility of three-dimensional models $[3,10]$. In many cases however, the methodology for producing the models is insufficiently detailed or relies on noncommercial hardware or proprietary software. Currently there is no straight-forward, nonproprietary method for converting crystallographic information framework (CIF) files into the stereo lithographic (STL) files required for recognition by three-dimensional printers. Such a method would allow for the creation of inexpensive and accurate models of the over 800,000 structures (as of October 2015) in the various crystal structure databases. This approach was inspired by the range of unusual geometries and symmetries encountered in our own research $[11,12]$. Herein we describe a method that uses freely available software for the conversion of crystallographic data to a format that allows for the rapid creation of inexpensive molecular models.

\section{Experimental Details}

2.1. Hardware Details. The majority of molecules contain atoms that when printed would be unsupported from below and require the use of support material during the printing process. Although there are currently many inexpensive three-dimensional printers, all the models described herein were produced on an Up! Plus 3D Printer that automatically calculates and prints the necessary support material. This printer is sold in the United States as an Afinia H-479 printer. One important limitation of the printer used herein is its inability to colour specific atoms by type. Nevertheless, the ability to observe coordination geometry and other important concepts is still greatly enhanced through the use of such models.

2.2. Software Details. The free Mercury software suite (Version 2.2 or higher) from the Cambridge Crystallographic Data Centre was used for visualization, analysis and modification of crystallographic information framework (CIF) file data [13]. This software is available for Mac, Windows and Linux operating systems. The free Visual Molecular Dynamics software package (Version 1.8.7. or higher) was used for visualization of the Protein Data Bank (PDB) files and for rendering the stereo lithographic data (STL) files [14]. This software is available for Mac, Windows, and Linux operating systems. The free Netfabb Studio Basic software package was used to invert unit normals and fix other minor errors ([15], accessed July 2013). This software is available for Mac, Windows, and Linux operating systems. All software used herein was used with the Windows operating system.

2.3. File Conversion and Model Printing. The workflow diagram for file conversion is outlined in Figure 1. Once a CIF file is chosen, it is opened in Mercury to assess the structure. If the structure is large, contains a large number of hydrogen atoms, or contains disordered atoms (vide infra), it may be necessary to edit the file in a text editor so as to optimize the size and complexity of intricate structures. Even the largest protein may be successfully rendered as a STL file showing individual atoms using this methodology, and the Afinia $\mathrm{H}$ series printer has successfully printed molecules with over one hundred atoms. Printed molecules where the atoms are $7 \mathrm{~mm}$ or larger and bonds are $3 \mathrm{~mm}$ and thicker are easily handled and immediately recognizable when compared with twodimensional representations. Printing models where individual atoms (e.g., models of proteins) are smaller than $3 \mathrm{~mm}$ in diameter and bonds are smaller than $2 \mathrm{~mm}$ in diameter are 


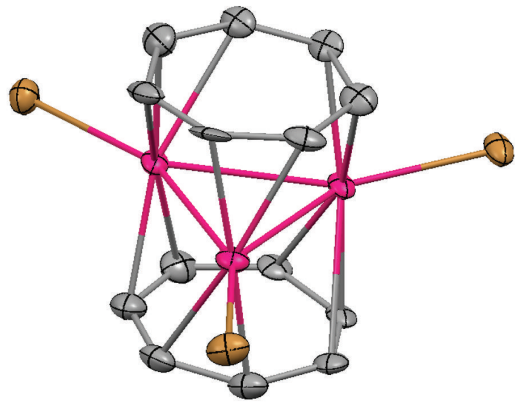

(a)

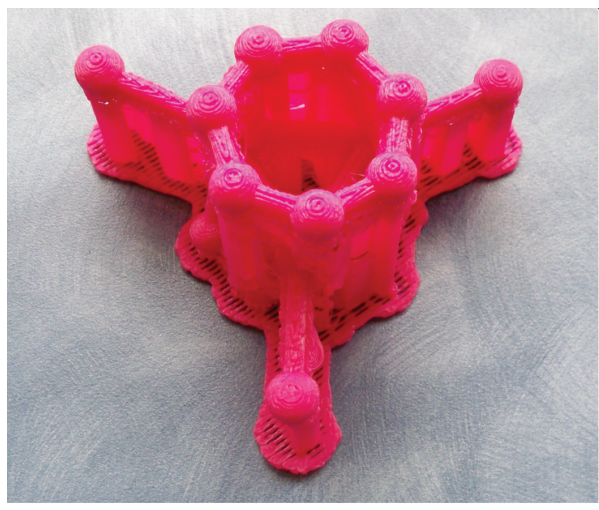

(c)

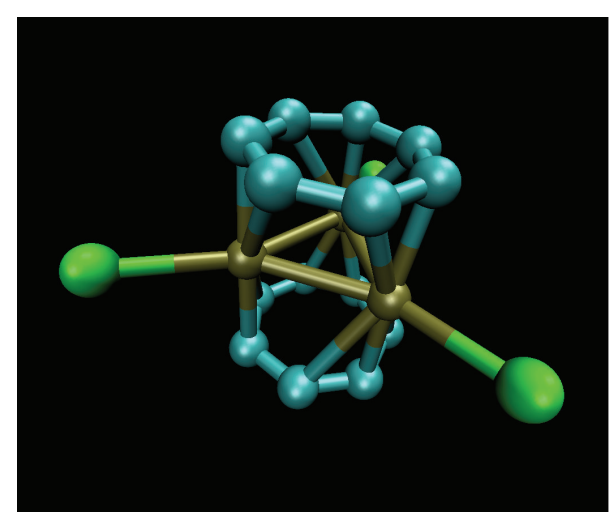

(b)

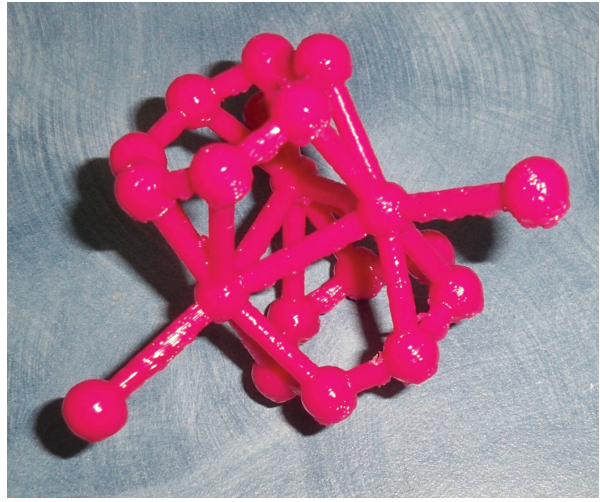

(d)

Figure 2: Conversion of CIF file data for $\left[\mathrm{PPh}_{4}\right]\left[\mathrm{Pd}_{3} \operatorname{Tr}_{2}\left(\mathrm{C}_{7} \mathrm{H}_{7}\right)_{2} \mathrm{Br}_{3}\right] \cdot \mathrm{MeCN}$ to a molecular model. (a) Thermal ellipsoid plot (50\% probability) as visualized in Mercury without hydrogen atoms, cation, and solvent atoms. (b) PDB file as visualized in VMD prior to rendering as a STL file. (c) Molecular model after printing showing attached support material. (d) Finished molecular model after removal of support material.

more challenging to remove from the accompanying support material. Minor molecules that are not directly attached to the main complex (e.g., solvate molecules or counterions) may be removed at this point if desired. The larger the molecule is, at this point, the smaller the final printed molecule will be due to the finite size of the printing platform. The Mercury software can then be used to save the crystal data in the PDB format. If the structure is already available in a PDB format, the above step is not necessary (e.g., protein structures). The new PDB file may then be opened using the VMD software package.

After the file is opened using VMD, the representation of the molecule may be modified to show the structure in different ways by changing the representations method. The CPK representation style is recommended for small molecules (molecular weight less than $1000 \mathrm{~g} \mathrm{~mol}^{-1}$ ) but not for very large structures (e.g., models of proteins). The size and resolution of the atoms and bonds may be modified at this point and the small molecule structures in the current article were saved using the following typical values (sphere scale $=1.0$, bond radius $=0.7$, sphere resolution $=30$, and bond resolution $=30$ ). At this point, the zoom option may be used to scale the model to an appropriate size. The above alterations are typically minor and serve mainly to improve the final resolution of the model. Once the model is optimized in VMD, the file must be rendered via the Render using STL option in order for it to be saved as a STL file. Other programs (e.g., PyMol, PMV, and VRML) are also capable of generating STL files, but the use of these programs was not investigated in the current work. The new STL file typically has errors which may be repaired using the Netfabb Studio Basic software package. Applying the "Part $\rightarrow$ Invert Normals" operation in Netfabb Studio Basic is essential for creating useable STL files, as the rendering process typically creates objects with the surface normals reversed. Holes and other defects in the STL mesh may be repaired using the "Heal $\rightarrow$ Default Repair" option. The STL file may then be opened in the native software supplied with the printer for any final adjustments (e.g., scaling, rotation) before the structure is printed by the three-dimensional printer. In some cases the triangular STL mesh may still have minor imperfections. Such a model may still print normally on most three-dimensional printers or in the case of the Up Afinia printer; the "Unsolid Model" software option will allow the model to print normally.

At this point, consideration for how the model will be printed may be taken into account. Specific parameters include how the model will be orientated on the build platform, the amount of empty interior space the model 


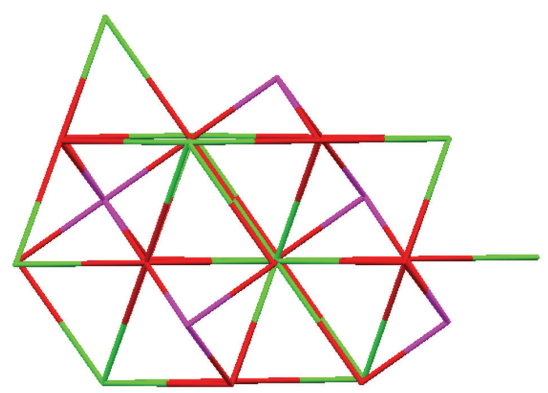

(a)

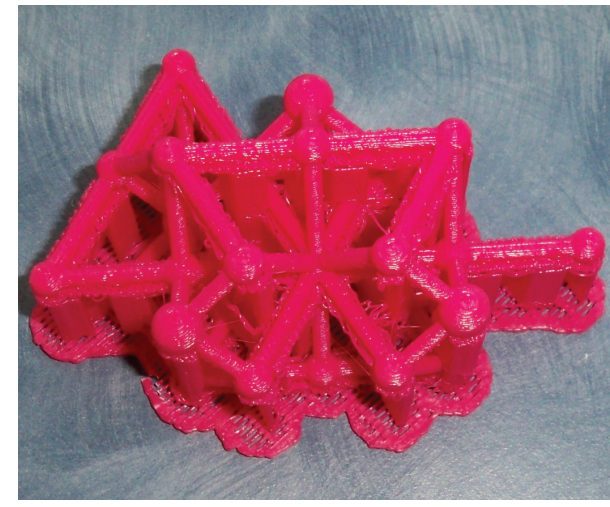

(c)

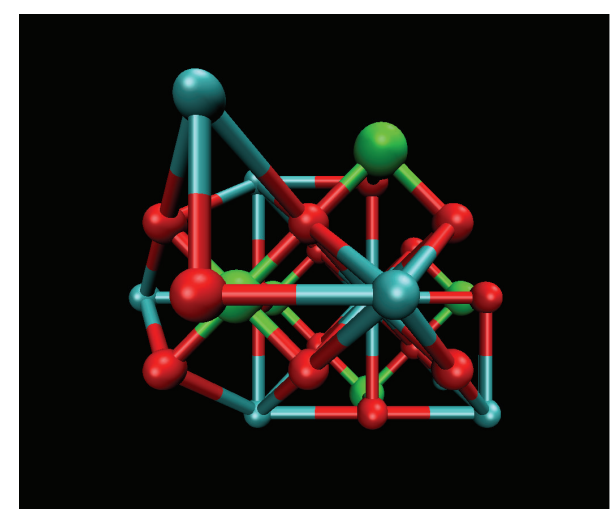

(b)

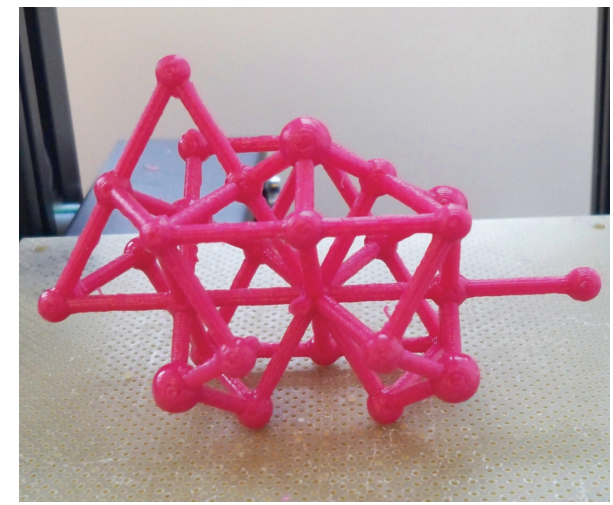

(d)

FIGURE 3: Conversion of CIF file data for $\mathrm{CaTiO}_{3}$ to a molecular model. (a) Thermal ellipsoid plot (90\% probability) as visualized in Mercury. (b) PDB file as visualized in VMD prior to rendering as an STL file. (c) Ball and stick molecular model after printing showing attached support material. (d) Finished molecular model on printer bed after removal of support material.

will contain (fill factor or fill ratio), and how much and at what overhang angle support material should be used. Such specific details are dependent upon the nature of the model to be printed. Support material is automatically calculated in the software and may be rapidly and easily removed after printing is complete. Models sufficient for repeated use in a class of thirty students can be printed in as little as a day and used repeatedly thereafter.

\section{Results and Discussion}

The use of the above method has worked with a high degree of success for the small organometallic molecules synthesized in our laboratory and these models serve as an excellent student recruitment tool. To confirm that our method could be applied generally, we selected a series of CIF files of different molecular types to confirm the workflow methodology outlined in Figure 1.

3.1. Molecular Model of $\left[\mathrm{PPh}_{4}\right]\left[\mathrm{Pd}_{3}\left(\mathrm{C}_{7} \mathrm{H}_{7}\right)_{2} \mathrm{Br}_{3}\right] \cdot \mathrm{MeCN}$. The complex $\left[\mathrm{PPh}_{4}\right]\left[\mathrm{Pd}_{3}\left(\mathrm{C}_{7} \mathrm{H}_{7}\right)_{2} \mathrm{Br}_{3}\right] \cdot \mathrm{MeCN}$ (Figure 2) possesses a number of unusual features which are not able to be recreated using standard molecular model kits, including metal-metal interactions and odd-numbered aromatic rings with eta bonding interactions [11]. Hydrogen atoms, solvate molecules, and the cation were removed manually from the text version of the CIF file under the atom_site_loop section. The time to convert the file from a text document to a CIF file, a PDB file, and then a repaired STL file was approximately ten minutes. The time to print the model was approximately ninety minutes and required no user intervention.

3.2. Molecular Model of $\mathrm{CaTiO}_{3}$. The perovskite crystal structure is educationally interesting and lends its name to the perovskite family of complexes with general formula $\mathrm{ABO}_{3}$. A CIF file for the calcium titanate perovskite was obtained from the American Mineralogist Crystal Structure Database [16] and was processed using the same methodology (Figure 1). The structure was successfully converted, rendered, and printed as a ball and stick structure to allow observation of the internal structure (Figure 3).

3.3. Molecular Model of Alpha-Carbonic Anhydrase. The Protein Data Bank contains over 100,000 molecular structures (as of May 2014) that are of interest to both crystallographers and chemists. The PDB file for alpha-carbonic anhydrase (1CA2.PDB) [17] was obtained from the Protein Data Bank and edited to remove several water molecules for clarity of 


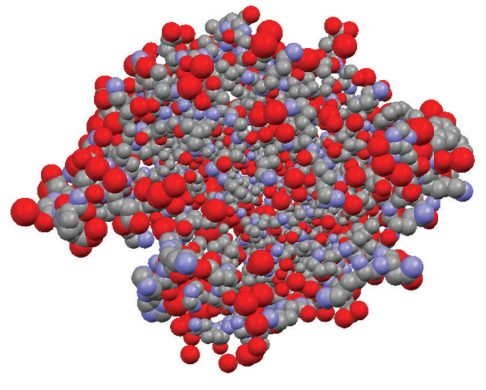

(a)

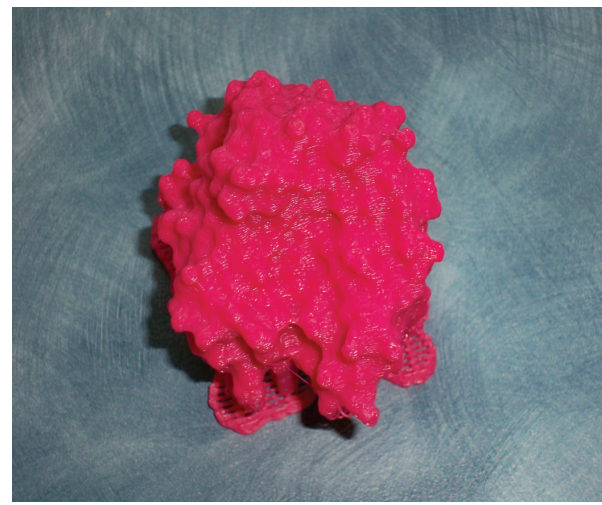

(c)

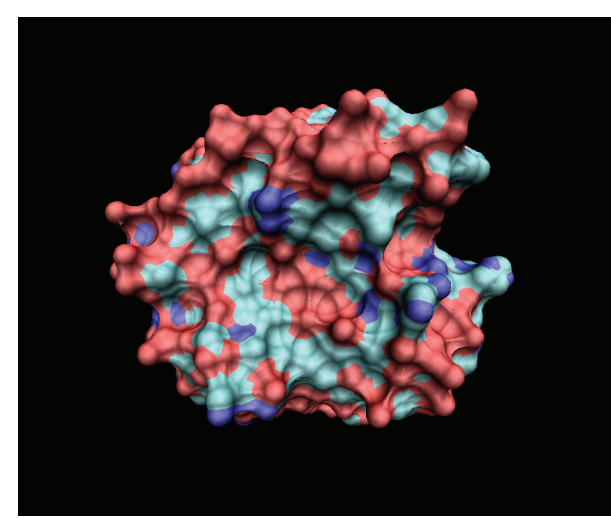

(b)

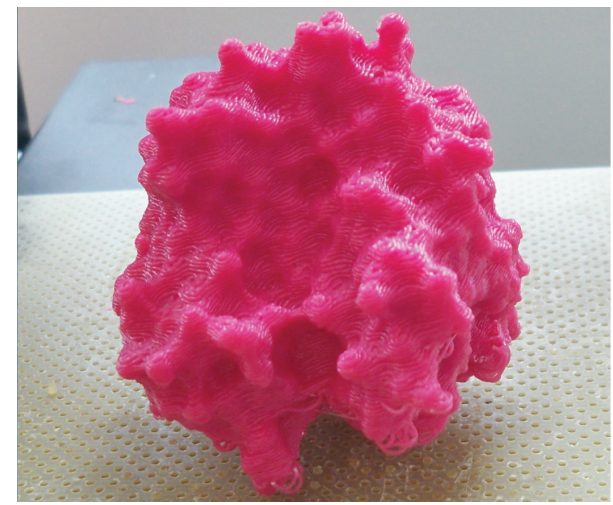

(d)

FIgure 4: Conversion of PDB file data for alpha-carbonic anhydrase to a molecular model. (a) Thermal ellipsoid plot (50\% probability) as visualized in Mercury without hydrogen atoms. (b) PDB file as visualized in VMD using SURF representation prior to rendering as an STL file. (c) Molecular model after printing showing attached support material. (d) Finished molecular model after removal of support material showing the active site in the cleft at the front of the model.

the overall structure. After opening the file in Mercury, it was judged that due to the large number of atoms it would not be possible to print the file with individually resolved atoms. Instead the protein was rendered in VMD using the SURF representation (Probe length $=2.0$ ) which generated a representation of the surface of the protein including the cleft in which the active site resides. After rendering and repairing of the structure, there were a small number of atoms $(<10)$ that were still improperly rendered, but the model was successfully printed through the use of the "Unsolid Model" printer option (Figure 4).

3.4. Molecular Models of Other Compounds. Using the above approach it was possible to promptly and accurately convert a series of CIF files [18-21] to their corresponding molecular models (Figure 5). Data conversion was rapid, typically taking less than fifteen minutes, and this method also allows for the printing of structures that show molecular disorder. The time necessary to print the models depended upon the size of the model, the density of the fill factor, the quality of the structure (resolution), and the amount of support, but in general printing times varied between one to three hours. Undergraduate chemistry students could be taught how to accomplish the file conversion during a single three-hour inorganic chemistry laboratory.

When printed at a suitably dense fill ratio (typically $\geq 90 \%$ ), the models are highly robust and resistant to damage. In the event that a model is damaged, repairs can be quickly carried out using acetone to rejoin the individual pieces of the printed model of $\left[\mathrm{Ru}_{2}(\mathrm{ap})_{4}\left(\mathrm{C} \equiv \mathrm{CSiMe}_{3}\right)\right]$ (ap = 2anilinopyridinate) [22] (see Figure 6). The orientation of bonds in the model, either parallel or perpendicular to the direction of the printer, does not significantly affect the strength of the resulting model. Molecules that exhibit molecular disorder can be printed with or without the disordered components or atoms. In the monohydroxyruthenocene complex $\left[\mathrm{Ru}\left(\eta^{5}-\mathrm{C}_{5} \mathrm{H}_{5}\right)\left(\eta^{5}-\mathrm{C}_{5} \mathrm{H}_{4} \mathrm{OH}\right)\right]$, the hydroxy group is disordered with occupancies of 0.5 due to the presence of a mirror plane [23]. Selection of just one of the disordered components through modification of the CIF file allows for the correct molecular form of the complex to be created (see Figure 7). 


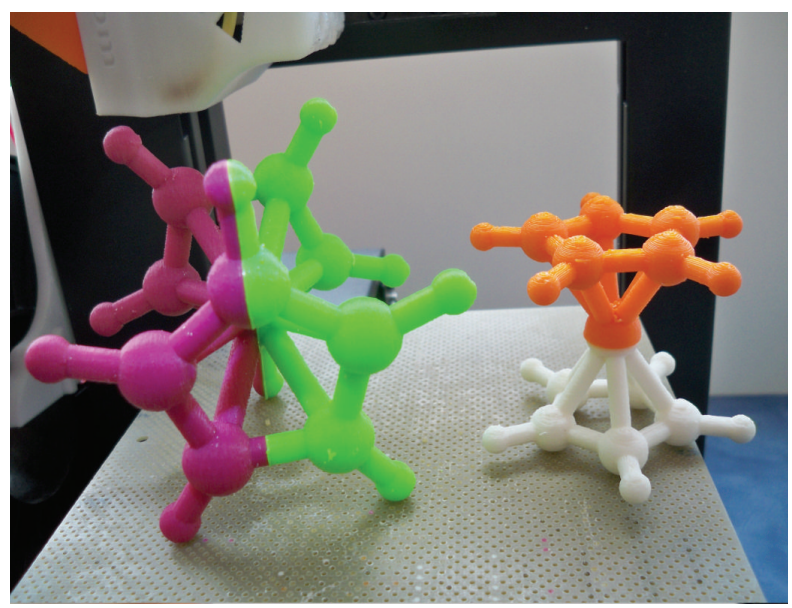

(a)

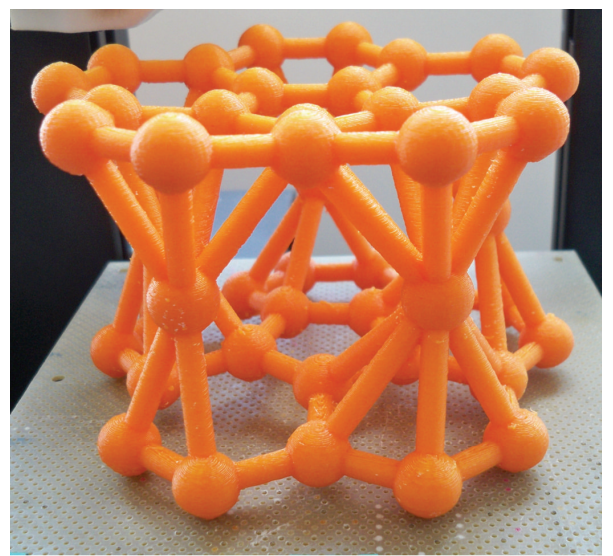

(c)

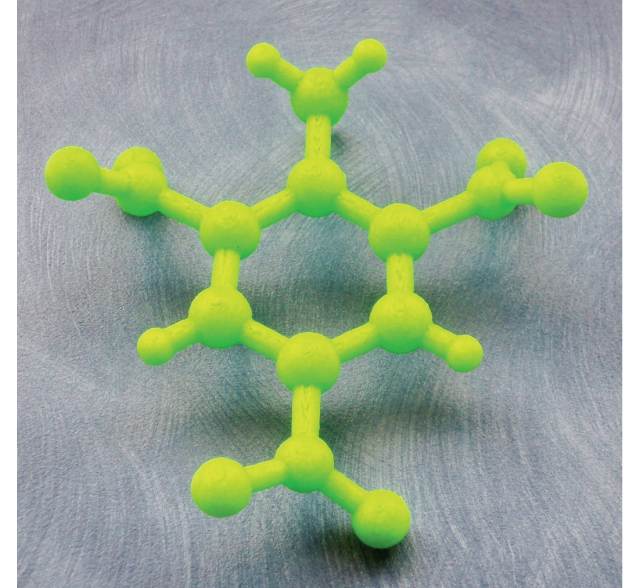

(b)

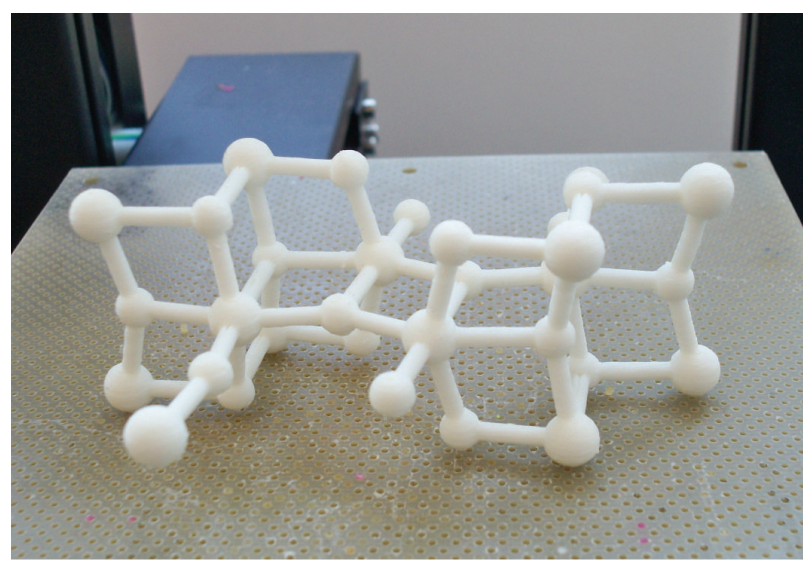

(d)

Figure 5: Molecular models generated by conversion of CIF file data. (a) Ferrocene. (b) 2,4,6-trinitrotoluene. (c) Lithium-coranullene sandwich complex. (d) $\mathrm{TiO}_{2}(\beta)$.

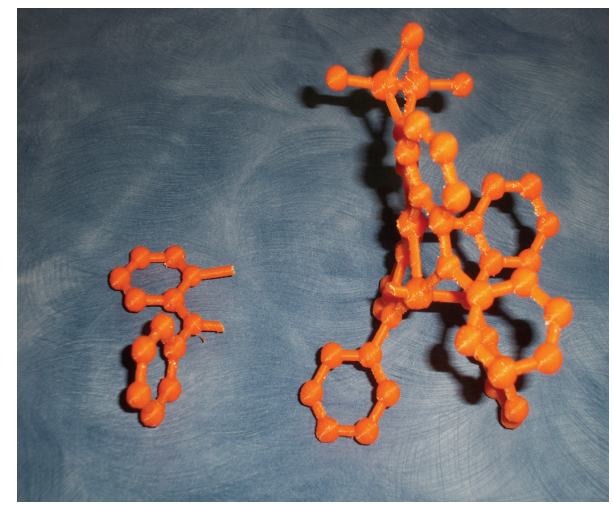

(a)

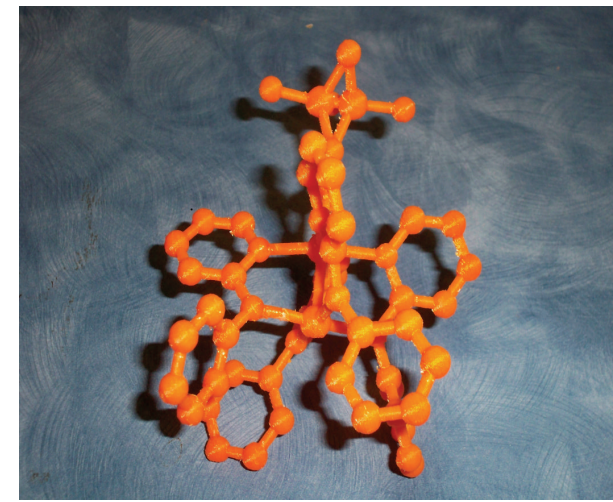

(b)

FIGURE 6: Repair of a molecular model made from ABS (Acrylonitrile-Butadiene-Styrene) plastic using acetone. 


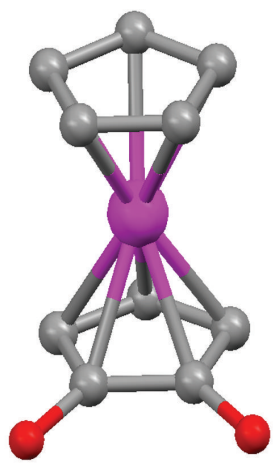

(a)

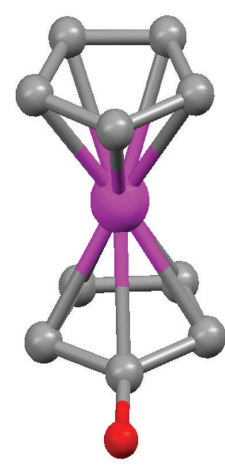

(c)

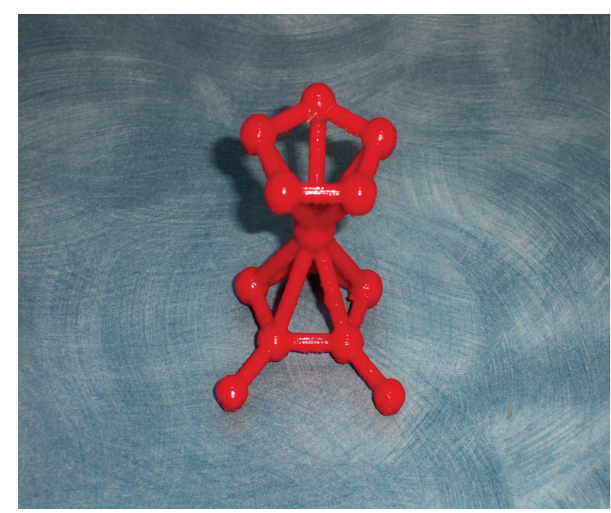

(b)

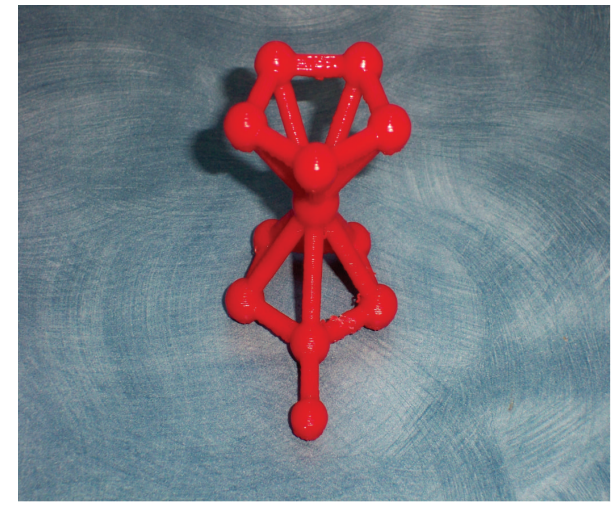

(d)

Figure 7: Thermal ellipsoid plot (50\% probability) as visualized in Mercury of hydroxy-ruthenocene $\left[\mathrm{Ru}\left(\eta^{5}-\mathrm{C}_{5} \mathrm{H}_{5}\right)\left(\eta^{5}-\mathrm{C}_{5} \mathrm{H}_{4} \mathrm{OH}\right)\right]$ showing virtual representations with either both disordered oxygen atom sites (a) or one of the disorder components removed (b). The resulting printed models ((c) and (d) resp.). Hydrogen atoms not displayed or printed.

\section{Summary and Conclusions}

Success in chemistry at the undergraduate level is strongly correlated with the ability to correctly visualize molecular structures. The ability to quickly and cheaply produce a threedimensional molecular model from CIF file data for use in the laboratory and the class room has been described. Current developments in three-dimensional printing are towards faster printing speeds, higher resolution, and lower start-up costs. Therefore, the ability to produce a large number of molecular models ranging from simple to complex will only become easier with time.

\section{Competing Interests}

The authors declare that they have no competing interests.

\section{Acknowledgments}

The authors thank Northern Arizona University (NAU) and the College of Engineering, Forestry and Natural Sciences (CEFNS) for start-up funding and NAU's Office of the Vice President for Research Faculty Grant Program for support. The authors thank the Research Corporation for Science
Advancement for their support through the Cottrell College Science Award (ID 10600) and the American Chemical Society Petroleum Research Fund (ACS-PRF Grant no. 51546-UR3). The authors wish to extend their thanks to Dr. Gary Nichol of the University of Edinburgh for fruitful discussions.

\section{References}

[1] S. R. Baker and L. Talley, "The relationship of visualization skills to achievement in freshman chemistry," Journal of Chemical Education, vol. 49, no. 11, pp. 775-776, 1972.

[2] M. E. Cass and H. S. Rzepa, "Visualizing metal tris chelates: visualizations to examine the structure and symmetry of metal tris chelates: symmetry operations, chirality, and mechanisms (bailar twist and rây-dutt) that racemize the $\Delta$ and $\Lambda$ Isomers," Journal of Chemical Education, vol. 85, no. 5, pp. 750-751, 2008.

[3] T. Herman, J. Morris, S. Colton et al., "Tactile teaching: exploring protein structure/function using physical models," Biochemistry and Molecular Biology Education, vol. 34, no. 4, pp. 247-254, 2006.

[4] T. Poon and R. Ovadia, "Using tactile learning aids for students with visual impairments in a first-semester organic chemistry course," Journal of Chemical Education, vol. 85, no. 2, pp. 240242, 2008. 
[5] C. A. Supalo, T. E. Mallouk, L. Rankel, C. Amorosi, and C. M. Graybill, "Low-cost laboratory adaptations for precollege students who are blind or visually impaired," Journal of Chemical Education, vol. 85, no. 2, pp. 243-247, 2008.

[6] D. C. Duffy, J. C. McDonald, O. J. A. Schueller, and G. M. Whitesides, "Rapid prototyping of microfluidic systems in poly(dimethylsiloxane)," Analytical Chemistry, vol. 70, no. 23, pp. 4974-4984, 1998.

[7] J. R. Roberts, E. Hagedorn, P. Dillenburg, M. Patrick, and T. Herman, "Physical models enhance molecular three-dimensional literacy in an introductory biochemistry course," Biochemistry and Molecular Biology Education, vol. 33, no. 2, pp. 105-110, 2005.

[8] S. K. Hurst, “Thingiverse, Octahedral atom model," October 2013, http://www.thingiverse.com/thing:4714.

[9] S. K. Hurst, Thingiverse: Square planar atom model, October 2013, http://www.thingiverse.com/thing:4715.

[10] M. A. Harris, R. F. Peck, S. Colton, J. Morris, E. Chaibub Neto, and J. Kallio, "A combination of hand-held models and computer imaging programs helps students answer oral questions about molecular structure and function: a controlled investigation of student learning," CBE-Life Sciences Education, vol. 8, no. 1, pp. 29-43, 2009.

[11] F. L. Mulligan, D. C. Babbini, I. R. Davis, S. K. Hurst, and G. S. Nichol, "Monomers and polymers of tripalladium $(0)$ ditropylium halides," Inorganic Chemistry, vol. 48, no. 7, pp. 2708-2710, 2009.

[12] D. C. Babbini, K. J. Cluff, N. B. Fisher, J. C. Charbonneau, G. S. Nichol, and S. K. Hurst, "New trimetallic sandwich complexes of platinum(0) and palladium(0)," Journal of Organometallic Chemistry, vol. 713, pp. 217-221, 2012.

[13] C. F. Macrae, P. R. Edgington, P. McCabe et al., "Mercury: visualization and analysis of crystal structures," Journal of Applied Crystallography, vol. 39, no. 3, pp. 453-457, 2006.

[14] W. Humphrey, A. Dalke, and K. Schulten, "VMD: visual molecular dynamics," Journal of Molecular Graphics, vol. 14, no. 1, pp. 33-38, 1996.

[15] Netfabb-Studio-Basic Netfabb Studio Basic, https://www.netfabb .com/products/netfabb-basic.

[16] T. Barth, "Die Kristallstruktur von Perowskit und verwandter Verbindungen," Norsk Geologisk Tidsskrift, vol. 8, pp. 201-216, 1925.

[17] A. E. Eriksson, T. A. Jones, and A. Liljas, "Refined structure of human carbonic anhydrase II at $2.0 \AA$ resolution," Proteins: Structure, Function and Genetics, vol. 4, no. 4, pp. 274-282, 1988.

[18] C. P. Brock and Y. Fu, "Rigid-body disorder models for the hightemperature phase of ferrocene," Acta Crystallographica Section $B$, vol. 53, no. 6, pp. 928-938, 1997.

[19] R. M. Vrcelj, J. N. Sherwood, A. R. Kennedy, H. G. Gallagher, and T. Gelbrich, "Polymorphism in 2-4-6 Trinitrotoluene," Crystal Growth and Design, vol. 3, no. 6, pp. 1027-1032, 2003.

[20] A. V. Zabula, A. S. Filatov, S. N. Spisak, A. Y. Rogachev, and M. A. Petrukhina, "A main group metal sandwich: five lithium cations jammed between two corannulene tetraanion decks," Science, vol. 333, no. 6045, pp. 1008-1011, 2011.

[21] T. Ishiguro, K. Tanaka, F. Marumo, M. G. Ismail, S. Hirano, and S. Sōmiya, "Freudenbergite," Acta Crystallographica Section B, vol. 34, no. 1, pp. 255-256, 1978.

[22] G. Zou, J. C. Alvarez, and T. Ren, "Ru- $\sigma$-alkynyl compounds of tetraanilinopyridinato-diruthenium(II,III) core: synthesis and structural characterization," Journal of Organometallic Chemistry, vol. 596, no. 1-2, pp. 152-158, 2000.
[23] K. Kirchner, K. S. Kwan, and H. Taube, "Synthesis, characterization, and crystal structure of hydroxyruthenocene," Inorganic Chemistry, vol. 32, no. 22, pp. 4974-4975, 1993. 

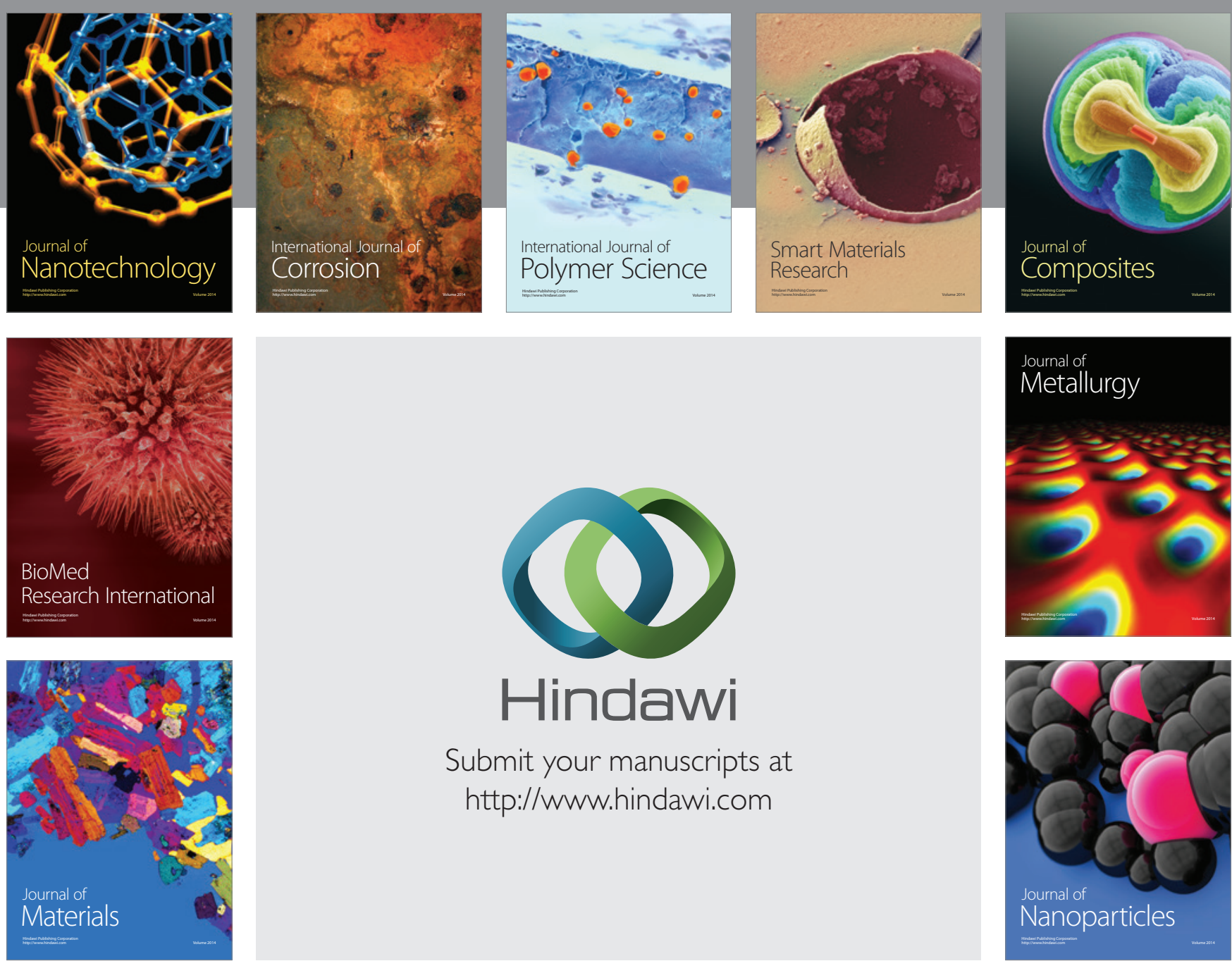

\section{Hindawi}

Submit your manuscripts at

http://www.hindawi.com

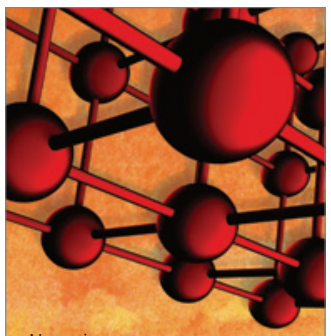

Materials Science and Engineering
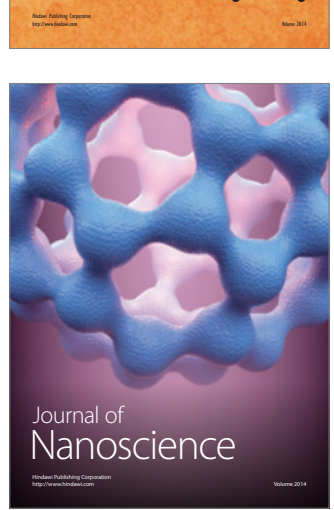
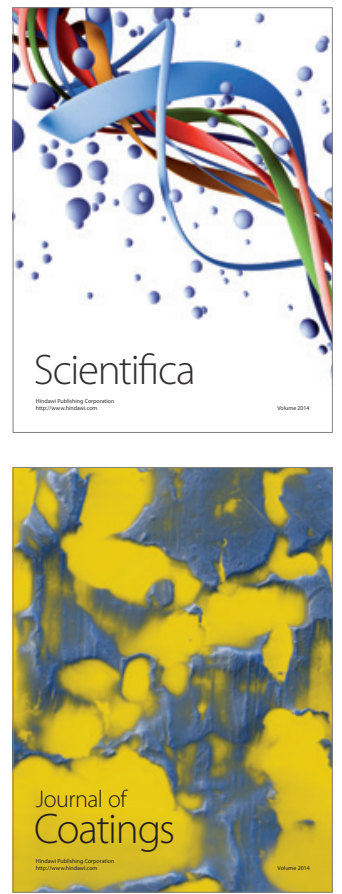
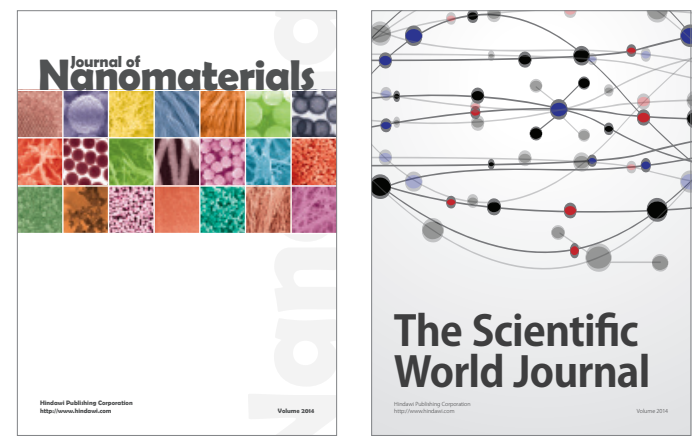

The Scientific World Journal
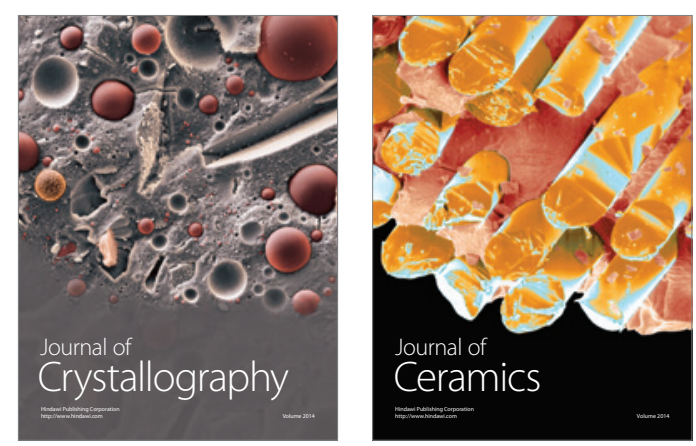
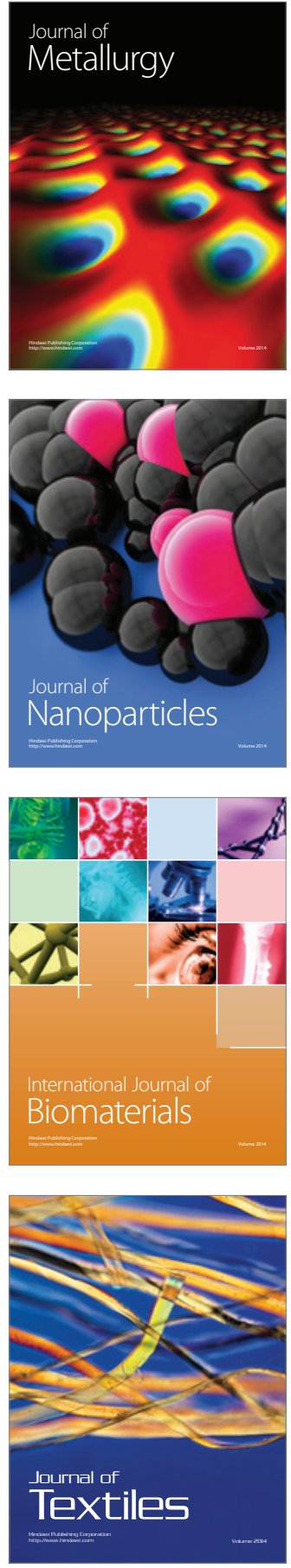